\title{
Magnetic Resonance Imaging of Arteriovenous Malformation of Pinna: A Case Report
}

\author{
Md. Hafizur Rahman, ${ }^{1}$ Nashid Amir, ${ }^{2}$ Md. Saiful Islam, ${ }^{3}$ AHM Tohurul Islam, ${ }^{4}$ Md. Anisur Rahman, ${ }^{5}$ \\ Md. Durrul Huda ${ }^{6}$
}

\begin{abstract}
Vascular malformations and tumors comprise a wide, heterogeneous spectrum of lesions that often present a diagnostic and therapeutic challenge. Auricular AVM is a rare entity \& needs MRI \& MR angiography for optimal diagnosis and proper pre \& post-operative evaluation and management. Very few such cases were reported in previous literatures. We present a rare case of auricular AVM in a 20 year old woman. MRI with MRA showed the lesion extension \& characteristics excellently \& also depicted the posterior auricular \& superficial temporal arteries to be the feeding vessels. Clinical suspicion of AVM was confirmed by imaging \& the patient was referred for surgical excision.

Key Words: Arteriovenous malformation (AVM), Magnetic resonance imaging (MRI), Magnetic resonance angiography (MRA), Vascular tumor, Feeding vessels, Nidus.
\end{abstract}

TAJ 2021; 34: No-2: 129-133

\section{Introduction}

Arteriovenous malformations (AVMs) are lesions of abnormal vascular development characterized by an abnormal communication between one or more arteries and veins without an intervening capillary bed. ${ }^{1}$ Vascular malformations are congenital diseases, may present at birth or later in life. Previously congenital diseases of vascular origin were classified as haemangioma and arteriovenous malformation. This classification has been modified over the years and now vascular anomalies are divided into vascular tumors and vascular malformations. Vascular malformations are then further subdivided on their dynamic flow characteristics into high-flow and low-flow vascular malformations, the two distinct groups having quite different management protocols. $^{2}$ AVMs are a common entity in intracranial location, but extracranial head-neck region AVMs are a much rare disease and poses a great challenge for the radiologists and surgeons in accurate diagnosis \& successful treatment. Patients with AVM in ear or neck region usually presents at childhood or early adult life with a palpable, firm, slow growing, reddish, non tender lesion with or without superficial ulceration \& bleeding. AVM of external ear is an uncommon disease and very few literatures have been published on this so far. Although clinically suspected, imaging plays a crucial role in the confirmation of arteriovenous malformations and preoperative planning. Color Doppler Ultrasonography, CT Angiogram (CTA)

1 Professor, Department of Radiology \& Imaging, Rajshahi Medical College.

2 Radiologist, Department of Radiology \& Imaging, Rajshahi Medical College Hospital.

${ }^{3}$ Associate Professor, Department of Radiology \& Imaging, Rajshahi Medical College.

${ }^{4}$ Associate Professor, Department of Radiology \& Imaging, Rajshahi Medical College.

${ }^{5}$ Assistant Professor, Department of Radiology \& Imaging, Rajshahi Medical College

${ }^{6}$ Assistant Professor, Department of Radiology \& Imaging, Rajshahi Medical College. 
\& MR Angiograms (MRA) are the key imaging modalities. The central nidus with feeding artery, draining venous channels \& associated vascular collaterals are well idenified in CTA \& MRA. Treatment options include surgical excision with or without preoperative endovascular embolization. Here we present a case of a 20 years old female patient with gradually enlarging non tender palpable mass in left ear who underwent contrast enhanced MRI \& MRA and was diagnosed as a case of AVM of left external ear.

\section{Case Report:}

A 20 year old lady presented to us with the complaints of gradually increasing swelling in her left ear for a year. On examination, the swelling was found to be pulsatile, lobulated, non tender, soft to firm, free from overlying skin. No overlying skin discoloration, ulceration, adjacent collaterals were seen. There was no history of pain, bleeding, tinnitus, difficulty in hearing. No definite audible thrill could be detected. External auditory canal and tympanic membrane were normal. Clinical diagnosis of high flow arterial malformation was made on history and examination by the clinicians. The patient was then advised to do a contrast enhanced MRI of brain with an MR angiogram of cerebral \& neck vessels. Non contrast MR revealed : an irregular tortuous tuft of vascular flow voids measuring about $5.1 \times 1.9 \times 5.0 \mathrm{~cm}$ (Anteroposterior $\mathrm{x}$ Transverse diameter $\mathrm{x}$ Craniocaudal) involving pinna \& posterior auricular region with relative sparing of the lobule on both T1WI \& T2WI. After intravenous Gadolinium administration, enlarged serpiginous structures showing flow-related enhancement in the left auricular and pinna region was demonstrated. The dilated vascular channels on MRA were from the major branches of left external carotid artery namely- posterior auricular artery \& auricular branches of superficial temporal artery (the feeding arteries) and adjacent dilated venous tributaries were the draining veins of the AVM nidus. No intracranial extension of the lesion, no adjacent bony or cartilage involvements were evident. MRI \& MRA depicted the AVM clearly which precluded further imaging.

\section{Pictorial Review:}

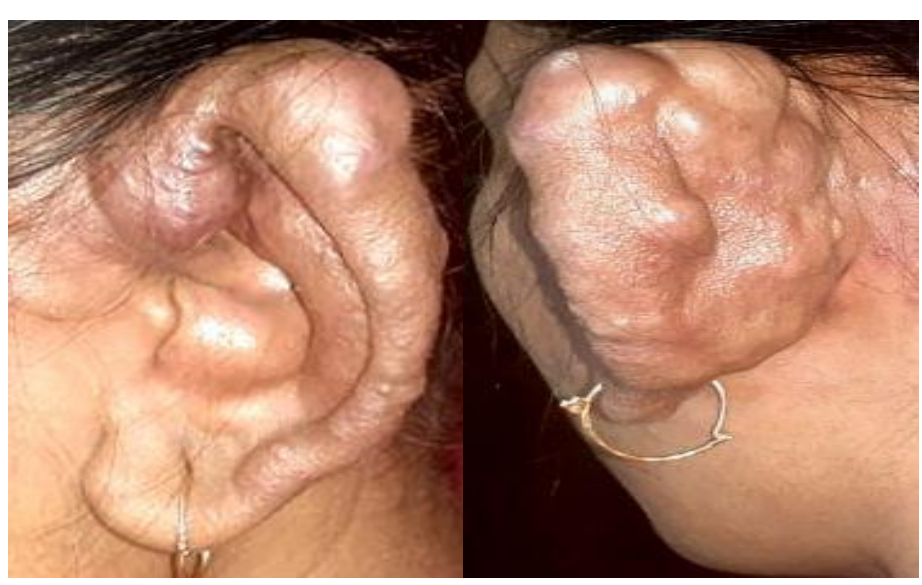

Figure 1 showing: Lobulated swelling in left pinna, lobule \& posterioe auricular region. No overlying skin ulceration, discoloration, collaterals are seen. 


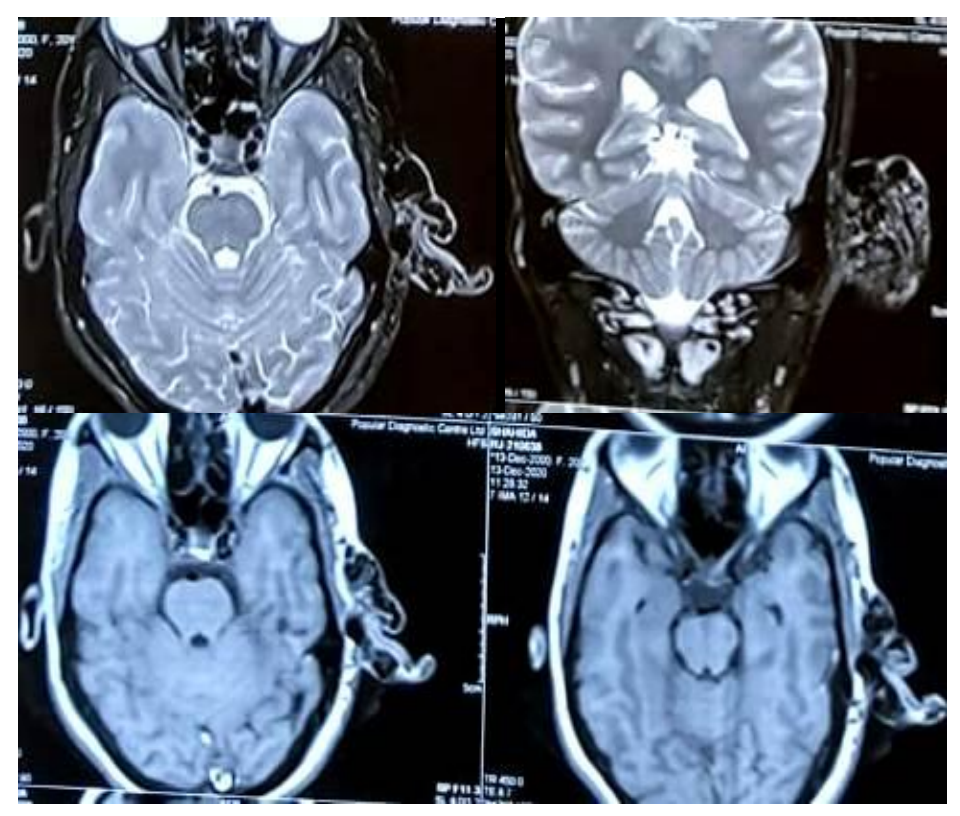

Figure 2:

Axial sections of T2WI \& pre contrast T1WI showing: multiple serpiginious vascular flow voids in left auricular region.

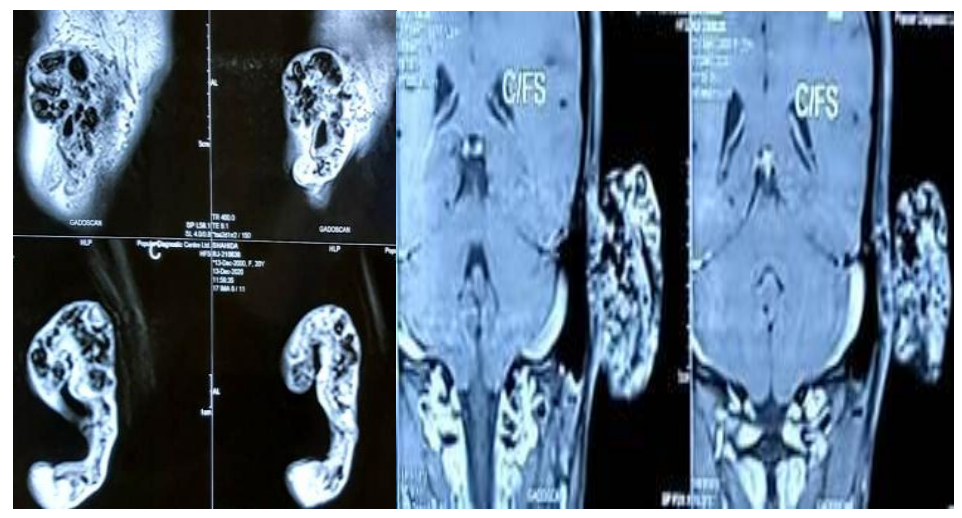

Figure 3:

Coronal sections of Post contrast T1WI showing: flow related enhancement of the AVM nidus in left auricular region. 


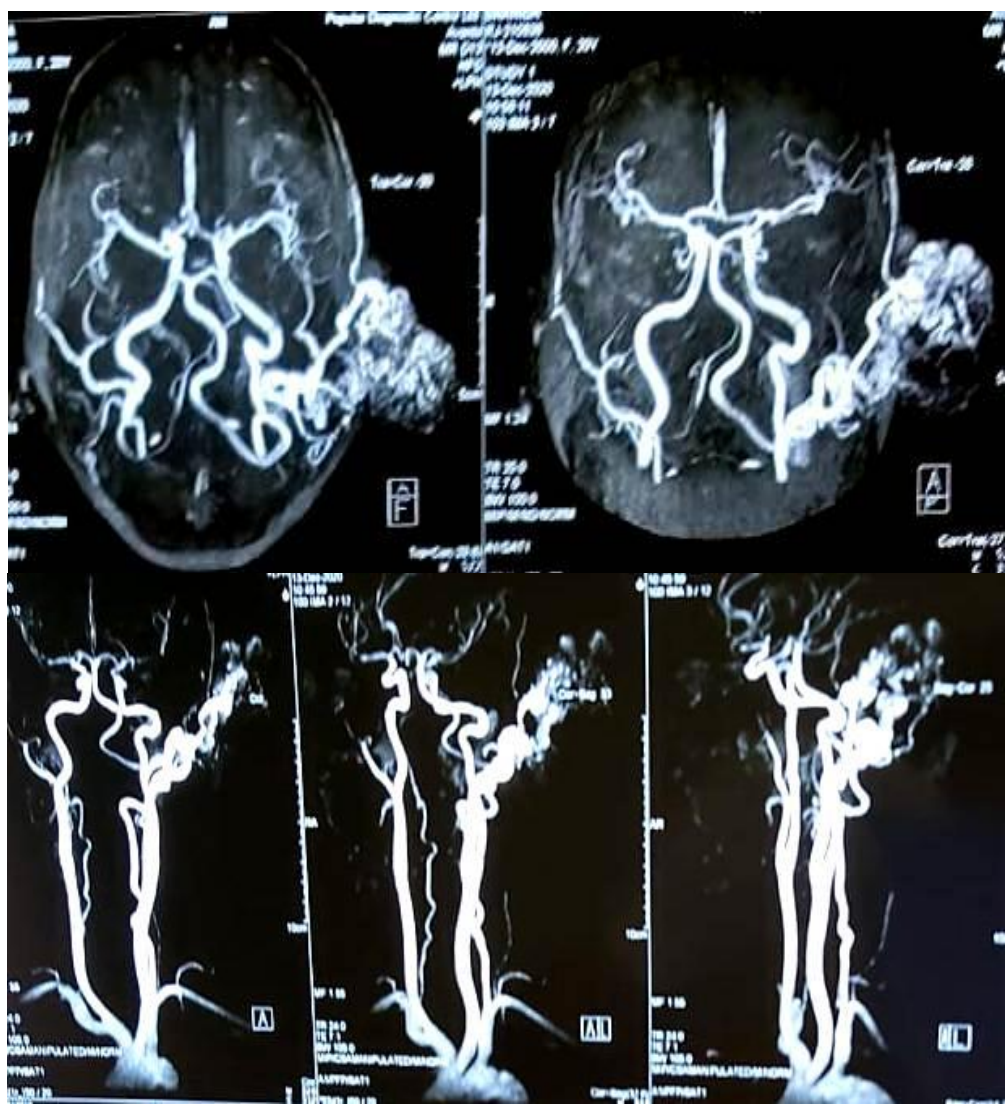

Figure 4:

Axial \& Coronal sections of Post contrast MRA showing: the AVM nidus being supplied by the posterior auricular \& auricular branches of superficial temporal arteries, both are branches of external carotid artery. Rapid shunting of contrast material in adjacent dilated venous channels confirms the diagnosis of AVM.

\section{Discussion}

Arteriovenous malformations are rare in the auricular region but are commonly found intracranially. AVM is composed of a central nidus with anomalous arteriovenous shunts and a network of surrounding collateral vessels. The short circuit or shunting between the high pressure arterial and low pressure venous system accounts for most of the clinical symptoms, anatomical changes, and progression of the lesions. ${ }^{6}$ The exact pathogenesis of an arteriovenous malformation (AVM) has not been clearly

defined. One theory postulates that these malformations arise due to error in foetal development during 4th to 6th gestation week as a result of the failure of regression of arteriovenous channels in the primitive retiform plexus. It has also been postulated that local ischemia plays a role in pathogenesis of AVMs. ${ }^{7}$ MRI is the most valuable imaging modality for classification of vascular anomalies because it accurately demonstrates their extension and their anatomic relationship to adjacent structures. A comprehensive assessment of vascular anomalies requires functional analysis of the involved vessels. Dynamic contrast enhanced MR angiography provides information about the hemodynamics of vascular anomalies and allows differentiation of high-flow and low-flow vascular malformations. Moreover, MR imaging is useful in assessment of successful treatment and establishment of a long-term management 
strategy. Preoperative assessment \& postoperative long term follow up are effectively done by MRI. The sequences commonly used in a vascular anomaly protocol include $\mathrm{T} 1$ spin-echo sequences and T2 fat-saturated and / or short inversion recovery (STIR) imaging to delineate the lesion from the surrounding fat and determine its relation to surrounding structures, particularly the airway and neurovascular bundles. Color Doppler Ultrasonography \& CT angiogrphy are two other modalities that play important role in AVM diagnosis \& management. CT scan is especially required if bony involvement or destruction is anticipated. However, the excellent soft tissue delineation \& absence of ionizing radiation makes MRI the modality of choice. Its non-invasiveness also is beneficial to the patient and precludes the necessity of DSA.

\section{Conclusion}

Vascular malformations and tumors comprise a wide, heterogeneous spectrum of lesions that often present a diagnostic and therapeutic challenge. MRA is a noninvasive excellent angiographic imaging modality of the craniofacial vascular lesions. The 3D MRA in particular with high spatial and temporal resolution provides distinct features that enables excellent lesion detection, characterization, visualization of feeding arteries and draining veins and complete extensions. It also allows the accurate differentiation of hemangiomas from AVMs that is sometimes difficult using clinical examination and Doppler Ultrasonography. MRI with adjunct MRA plays an important role in extension evaluation, treatment planning and follow up.

\section{References}

1. Meher, R., Varshney, S. and Pant, H.C. (2008) Arteriovenous Malformation Related to the Pinna. Hong Kong Medical Journal, 14, 157-159.

2. Jackson IT, Carreno R, Potparic Z, Hussain K. Hemangiomas, vascular malformations, and lymphovenous malformations: classification and methods of treatment. Plast Reconstr Surg 1993;91:1216-30

3. McCafferty IJ, Jones RG. Imaging and management of vascular malformations. Clin Radiol 2011;66:1208-18.

4. Rosenblatt M. Endovascular management of venous malformations. Phlebology 2007;22:264-275.

5. Legiehn GM, Heran MK. Classification, diagnosis and interventional radiologic management of vascular malformations. Orthop Clin North Am 2006;37:435474.

6. Bijendar KM, Sweta M, Abhishek G, Meena ML, Baser SC. A rare case of arteriovenous malformation of the pinna and review of literature. Journal of Medical Science and Clinical Research 2013 ;Volume:1:Issue:2;Pages107-111;.

7. Gupta A, Gupta S, Kumar A, Bhattacharaya S, Jha M, Tiwari V. High- Flow Vascular Malformation of Ear: A Case Report. World J Plast Surg 2018;7(2):256-260. 